\title{
EXISTENCE OF SOLUTIONS IN A CONE FOR NONLINEAR ALTERNATIVE PROBLEMS
}

\author{
JUAN J. NIETO
}

\begin{abstract}
Using the alternative method we present sufficient conditions for the existence of positive solutions to nonlinear equations at resonance and extend a well-known result of Cesari and Kannan.
\end{abstract}

Introduction. Cesari and Kannan [2] proved an abstract result in terms of the alternative method. Their result and some of its ramifications (see [1]) have been applied to a large class of problems at resonance to prove the existence of solutions.

Let $E$ be a Banach space. We say that $C$ is a cone in $E$ if $C$ is a nonempty, convex subset of $E$ such that $\lambda C \subset C$ for every $\lambda \geqslant 0$.

Here we prove the existence of solutions in a cone for equations at resonance of the form $L u=N u$, where $L$ is a linear operator and $N$ is a (nonlinear) operator. In the case when the cone is $E$, we obtain the well-known result of Cesari and Kannan [2].

In applications, for instance, if $L$ is an elliptic operator on a bounded domain $\Omega$ of $\mathbf{R}^{n}$, one usually takes $E$ as a subspace of $L^{2}(\Omega)$ and the cone $C=\{u \in E: u \geqslant 0$ a.e. in $\Omega$.

Also, our result is related to that of Gaines and Santanilla [3] concerning the existence of solutions in a convex set.

Main result. Let $E$ and $F$ be Banach spaces with norms \|\|$_{E}$ and \|\|$_{F}$, respectively. Let $L: D(L) \subset E \rightarrow F$ be a linear operator and $N: E \rightarrow F$ a continuous (nonlinear) operator such that $N$ maps bounded sets into bounded sets. Assume that $C$ is a cone in $E$ and

there exists a continuous map $\gamma: E \rightarrow C$ such that $\gamma(c)=c$ for every $c \in C$, and $\gamma$ maps bounded sets in $E$ into bounded sets in $E$.

In addition, suppose that $L$ is a Fredholm map of index 0 and there exist projections $P: E \rightarrow E, Q: F \rightarrow F$, and a linear map $H:(I-Q) F \rightarrow(I-P) E$

Received by the editors February 16, 1984 and, in revised form, June 17, 1984.

1980 Mathematics Subject Classification. Primary 47H15, 34B15, 34C15, 35G30, 35J40. 
satisfying

$$
\begin{array}{ll}
H(I-Q) L u=(I-P) u & \text { for every } u \in D(L) \\
Q L u=L P u & \text { for every } u \in D(L) \\
L H(I-Q) N u=(I-Q) N u & \text { for every } u \in E
\end{array}
$$

Thus, it is well known that $L u=N u$ is equivalent to the coupled system of equations

$$
\begin{array}{ll}
Q N u=0 & \text { (bifurcation equation), } \\
u=P u+H(I-Q) N u & \text { (auxiliary equation). }
\end{array}
$$

We can write the spaces $E$ and $F$ as the direct sums $E=E_{0} \oplus E_{1}, F=F_{0} \oplus F_{1}$, where $E_{0}=P E, E_{1}=(I-P) E, F_{0}=Q F$, and $F_{1}=(I-Q) F$. Also, we assume

(3) $E_{0}=\operatorname{Ker} L, F_{1}=\operatorname{Im} L, D(H)=\operatorname{Im} L$ and $\operatorname{Im} H=E_{1} \cap D(L)$.

(4) $\operatorname{dim} E_{0}=\operatorname{dim} F_{0}<+\infty$.

(5) $H$ is completely continuous.

(6) There exist continuous maps $B: E \times F \rightarrow \mathbf{R}$ and $J: F_{0} \rightarrow E_{0}$ such that $B$ is bilinear, $J$ is one-to-one and onto, and

(i) for $v_{0} \in F_{0}, v_{0}=0$ iff $B\left(u_{0}, v_{0}\right)=0$ for all $u_{0} \in E_{0}$,

(ii) $B\left(J v_{0}, v_{0}\right) \geqslant 0$ for every $v_{0} \in F_{0}$ and $B\left(J v_{0}, v_{0}\right)=0$ iff $v_{0}=0$,

(iii) $J v_{0}=0$ iff $v_{0}=0$,

(iv) $B\left(u_{0}, J^{-1} u_{0}\right)=0$ iff $u_{0}=0$,

(v) $B\left(u_{0}, v_{0}\right)=B\left(J v_{0}, J^{-1} u_{0}\right)$ for every $u_{0} \in E_{0}, v_{0} \in F_{0}$.

REMARK. If $E \subset F$ and $F$ is a Hilbert space with inner product $\langle u, v\rangle$, then one can define $B\left(u_{0}, v_{0}\right)=\left\langle u_{0}, v_{0}\right\rangle$. Thus, if $F=L^{2}(\Omega)$,

$$
B\left(u_{0}, v_{0}\right)=\int_{\Omega} u_{0}(x) \cdot v_{0}(x) d x
$$

For $u \in E$ we write $u=u_{0}+u_{1}$, with $u_{0} \in E_{0}, u_{1} \in E_{1}$. With this, the auxiliary and bifurcation equations become $Q N\left(u_{0}+u_{1}\right)=0$ and $u_{1}=H(I-Q) N\left(u_{0}+u_{1}\right)$, respectively. We are now in a position to prove our result.

TheOREM. Let conditions (1)-(6) hold. In addition, assume there exists

(7) $J_{0}>0$ such that $\|N u\| \leqslant J_{0}$ for every $u \in C$,

(8) $R_{0}>0$ such that $B\left(u_{0}, Q N(u)\right) \leqslant 0$ for every $u=u_{0}+u_{1} \in C$, with $\left\|u_{0}\right\|=R_{0}$ and $u_{1}=H(I-Q) N\left(u_{0}+u_{1}\right)$, and

(9) $r_{0} \geqslant\|H(I-Q)\| \cdot J_{0}$ such that $(P+J Q N) \gamma u \in C$ and $H(I-Q) N \gamma(u) \in C$ for every $u \in S$, where

$$
S=\left\{u=u_{0}+u_{1} \in E:\left\|u_{0}\right\| \leqslant R_{0},\left\|u_{1}\right\| \leqslant r_{0}\right\} .
$$

Then $L u=N u$ has at least one solution $u \in S \cap C$.

Proof. The set $S$ is closed, bounded, and convex. Define the homotopy $T$ : $[0,1] \times S \rightarrow E$ by $T(\lambda, u)=\lambda P \gamma(u)+H(I-Q) N \gamma(u)+\lambda J Q N \gamma(u)$. Note that $T(\lambda, \cdot)$ is compact for every $\lambda \in[0,1]$ since $P$ and $Q$ are projections with finitedimensional range and $H$ is compact. For $\lambda=0, T(0, u)=H(I-Q) N \gamma(u) \in E_{1}$. 
Thus, by (9),

$$
\|T(0, u)\| \leqslant\|H(I-Q)\| \cdot\|N \gamma(u)\|<r_{0},
$$

which shows that $T(0, \partial S) \subset S$.

We shall now prove that $T(\lambda, u) \neq u$ for every $(\lambda, u) \in[0,1) \times \partial S$. Indeed, let $T(\lambda, u)=u$ and, consequently,

$$
\begin{gathered}
u_{0}=\lambda P \gamma(u)+\lambda J Q N \gamma(u), \\
u_{1}=H(I-Q) N \gamma(u) .
\end{gathered}
$$

If $u \in \partial S$, then either $\left\|u_{1}\right\|=r_{0}$ or $\left\|u_{0}\right\|=R_{0}$. In the first case, using (11), we get

$$
r_{0}=\left\|u_{1}\right\|=\|H(I-Q) N \gamma(u)\|<r_{0},
$$

which is a contradiction.

In the second case, $\left\|u_{0}\right\|=R_{0}$. Hence, by (9), $(P+J Q N) \gamma(u) \in C$ and $u_{0} \in C$ since $C$ is a cone. Also by (9), $u_{1}=H(I-Q) N \gamma(u) \in C$ and, consequently, $u=u_{0}+u_{1} \in C$. This implies, by the property of $\gamma$, that $\gamma(u)=u$ and $u_{0}=\lambda P u+$ $\lambda J Q N u$. This last inequality is equivalent to $(1-\lambda) u_{0}=\lambda J Q N u$. We assume that $\lambda>0$ since $\lambda=0$ implies $(1-\lambda) u_{0}=0$ and $u_{0}=0$. Thus, by (8) and (11), $B\left(u_{0}, Q N \gamma(u)\right) \leqslant 0$. On the other hand,

$$
\lambda B\left(u_{0}, Q N u\right)=\lambda B\left(J Q N u, J^{-1} u_{0}\right)=B\left((1-\lambda) u_{0}, J^{-1} u_{0}\right)>0,
$$

which is again a contradiction. Therefore, $T(\lambda, u) \neq u$ for every $(\lambda, u) \in[0,1) \times \partial S$, and we can conclude [4, Theorem 4.4.11] that $T(1, \cdot)$ has a fixed point. Hence, there exists $u \in S$ satisfying

$$
u=P \gamma(u)+H(I-Q) N \gamma(u)+J Q N \gamma(u) .
$$

Reasoning as before, $u \in C$ and satisfies the auxiliary and bifurcation equations, that is, $u$ is a solution of $L u=N u$ such that $u \in S \cap C$. This completes the proof of the Theorem.

If $C=E$ we obtain the result of [2].

COROLlARY. Let conditions (1)-(6) hold. In addition, assume there exists

(7)' $J_{0}>0$ such that $\|N u\| \leqslant J_{0}$ for every $u \in E$,

(8)' $R_{0}>0$ such that $B\left(u_{0}, Q N u\right) \leqslant 0$ for every $u=u_{0}+u_{1} \in E$, with $\left\|u_{0}\right\|=R_{0}$ and $u_{1}=H(I-Q) N\left(u_{0}+u_{1}\right)$.

Then $\mathrm{Lu}=\mathrm{Nu}$ has at least one solution.

For some particular cases of our result and applications to nonlinear boundary value problems, see [5].

ACKNOWLEDGEMENT. The author is thankful to the referee for helpful comments.

\section{REFERENCES}

1. L. Cesari, Functional analysis, nonlinear differential equations and the alternative method, Nonlinear Functional Analysis and Differential Equations (L. Cesari, R. Kannan and J. Schuur, eds.), Dekker, New York, 1976, pp. 1-197. 
2. L. Cesari and R. Kannan, An abstract theorem at resonance, Proc. Amer. Math. Soc. 63 (1977), 221-225.

3. R. E. Gaines and M. Santanilla, $A$ coincidence theorem in convex sets with applications to periodic solutions of ordinary differential equations, Rocky Mountain J. Math. 12 (1982), 669-678.

4. N. G. Lloyd, Degree theory, Cambridge Univ. Press, London and New York, 1978.

5. J. J. Nieto, Positive solutions of operator equations, preprint 1984.

Departamento de Teoria de Funciones, Facultad de Matemáticas, Universidad de Santiago, Santiago, Spain 